\title{
Quantifying Losses in Open-Circuit Voltage in Solution-Processable Solar Cells
}

\author{
Jizhong Yao, ${ }^{1, *}$ Thomas Kirchartz, ${ }^{2,3}$ Michelle S. Vezie, ${ }^{1}$ Mark A. Faist, ${ }^{1,4}$ Wei Gong, ${ }^{1,5}$ Zhicai He, ${ }^{6}$ \\ Hongbin Wu, ${ }^{6}$ Joel Troughton, ${ }^{7}$ Trystan Watson, ${ }^{7}$ Daniel Bryant, ${ }^{4}$ and Jenny Nelson ${ }^{1, \dagger}$ \\ ${ }^{1}$ Department of Physics and Centre for Plastic Electronics, Imperial College London, \\ South Kensington Campus SW7 2AZ, United Kingdom \\ ${ }^{2}$ IEK5-Photovoltaics, Forschungszentrum Jülich, 52425 Jülich, Germany \\ ${ }^{3}$ Faculty of Engineering and CENIDE, University of Duisburg-Essen, Carl-Benz-Straße 199, \\ 47057 Duisburg, Germany \\ ${ }^{4}$ Department of Chemistry and Centre for Plastic Electronics, Imperial College London, \\ South Kensington SW7 2AZ, United Kingdom \\ ${ }^{5}$ Key Laboratory of Luminescence and Optical Information, Ministry of Education \\ and Institute of Optoelectronics Technology, Beijing Jiao tong University, \\ Beijing 100044, People's Republic of China \\ ${ }^{6}$ Institute of Polymer Optoelectronic Materials and Devices, \\ State Key Laboratory of Luminescent Materials and Devices, \\ South China University of Technology, Guangzhou 510640, People's Republic of China \\ ${ }^{7}$ SPECIFIC, College of Engineering, Swansea University, Baglan Bay Innovation, Knowledge Centre, \\ Central Avenue, Baglan SA12 7AX, United Kingdom \\ (Received 7 January 2015; revised manuscript received 14 April 2015; published 28 July 2015)
}

\begin{abstract}
The maximum open-circuit voltage of a solar cell can be evaluated in terms of its ability to emit light. We herein verify the reciprocity relation between the electroluminescence spectrum and subband-gap quantum efficiency spectrum for several photovoltaic technologies at different stages of commercial development, including inorganic, organic, and a type of methyl-ammonium lead- halide $\mathrm{CH}_{3} \mathrm{NH}_{3} \mathrm{PbI}_{3-x} \mathrm{Cl}_{x}$ perovskite solar cells. Based on the detailed balance theory and reciprocity relations between light emission and light absorption, voltage losses at open circuit are quantified and assigned to specific mechanisms, namely, absorption edge broadening and nonradiative recombination. The voltage loss due to nonradiative recombination is low for inorganic solar cells $(0.04-0.21 \mathrm{~V})$, while for organic solar cell devices it is larger but surprisingly uniform, with values of $0.34-0.44 \mathrm{~V}$ for a range of material combinations. We show that, in $\mathrm{CH}_{3} \mathrm{NH}_{3} \mathrm{PbI}_{3-x} \mathrm{Cl}_{x}$ perovskite solar cells that exhibit hysteresis, the loss to nonradiative recombination varies substantially with voltage scan conditions. We then show that for different solar cell technologies there is a roughly linear relation between the power conversion efficiency and the voltage loss due to nonradiative recombination.
\end{abstract}

DOI: 10.1103/PhysRevApplied.4.014020

\section{INTRODUCTION}

In an ideal solar cell, the short-circuit current $J_{\mathrm{SC}}$ is determined by the absorbed photon flux, while the opencircuit voltage $V_{\mathrm{OC}}$ depends on the balance between photogeneration and recombination. This balance depends strongly on the band gap of the solar cell that determines both the light absorption and the flux of the recombining charge carrier. The Shockley-Queisser (SQ) limit [1] is one way to define an ideal solar cell and is based on the principle that any light-absorbing medium must also emit light, implying that radiative recombination is unavoidable. Thus, by calculating absorption and emission due to

\footnotetext{
* Corresponding author. jizhong.yao11@imperial.ac.uk

${ }^{\dagger}$ Corresponding author. jenny.nelson@imperial.ac.uk
}

radiative recombination, it is possible to determine the maximum open-circuit voltage $V_{\mathrm{OC}, \mathrm{SQ}}$ of an idealized device absorbing all light above a certain band-gap energy [2,3]. The resulting open-circuit voltage in the SQ limit depends on the intensity of incoming light, but, for the normal reference point of one sun illumination and no concentration, $q V_{\mathrm{OC}, \mathrm{SQ}}$ is reduced relative to the band gap by about $250 \mathrm{meV}$ for the typical range of band gaps used in photovoltaics [4]. Here $q$ is the elementary charge. For real solar cells, the open-circuit voltage is further reduced due to additional, nonradiative recombination that is neglected in the SQ theory or by a gradual absorption onset rather than the ideal step function assumed in the SQ limit.

Both sources of additional voltage loss are relevant to many solution-processable solar cells. In organic heterojunction solar cells, for instance, the emission usually originates from the charge transfer state at the donoracceptor interface [5], which is usually substantially 
redshifted relative to the absorption onset [6]. In addition, in organic solar cells, nonradiative recombination exceeds the amount of radiative recombination typically by a factor of $10^{6}$ or more, enhancing the difference between the absorption onset and the actual open-circuit voltage of a solar cell [7]. In order to maximize $V_{\mathrm{OC}}$ and reduce the difference between the ideal and actual cases, it is important to be able to quantify and distinguish the losses.

One frequently used way to quantify the losses in $V_{\mathrm{OC}}$ is to relate them to characteristic energy levels in the device such as the band gap in the case of inorganic solar cells [8-11] or to the charge transfer state energy in the case of organic heterojunction solar cells [5,12-14]. An alternative approach is to use a slightly extended version of the SQ theory where a radiative open-circuit voltage $V_{\mathrm{OC} \text {,rad }}$ that respects the actual light absorption spectrum is defined [15-17]. This allows us to assign the difference between $V_{\mathrm{OC}, \mathrm{SQ}}$ and $V_{\mathrm{OC}, \text { rad }}$ to the shape of the absorptance, which is influenced, for instance, by the band offsets at the donoracceptor heterointerface, and the difference between $V_{\mathrm{OC}, \text { rad }}$ and $V_{\mathrm{OC}}$ to nonradiative recombination [7,18-20].

In this work, we use electroluminescence and quantum efficiency data to determine $V_{\mathrm{OC}, \mathrm{SQ}}$ and $V_{\mathrm{OC} \text {,rad }}$ in order to explain the origin of losses in open-circuit voltage for a series of different solar cell technologies. We measure a series of solution-processable organic and perovskite solar cells and compare the obtained values with previously published data on different inorganic solar cell technologies. While most inorganic and perovskite solar cells show voltage losses due to nonradiative recombination, in organic solar cells the relatively high nonradiative losses are accompanied by additional losses due to the energy of photon emission being shifted to much lower energies relative to the onset of absorption. However, we identify organic materials such as poly(diketopyrrolopyrroleterthiophene) (PDPP3T) [21] where these losses due to the band offsets at the donor-acceptor interface are small and where the voltage losses come close to those of the perovskite solar cell we study.

Finally, we compare the losses due to nonradiative recombination with device efficiency and show that, over a wide range of efficiencies for inorganic and perovskite devices, there exists a roughly linear but fully empirical relation. In the case of organic solar cells, some devices, for instance, thieno[3,4-b]thiophene/benzodithiophene:[6,6]phenyl C71-butyric acidmethyl ester (PTB7: $\mathrm{PC}_{71} \mathrm{BM}$ ) solar cells [22], still roughly follow the linear trend, while most organic systems deviate from the trend in terms of efficiency.

\section{RADIATIVE LIMIT TO THE OPEN-CIRCUIT VOLTAGE}

The open-circuit voltage is the voltage where recombination current $J_{\text {rec }}$ and photocurrent $J_{\mathrm{ph}}$ are equal, i.e., where

$$
J_{\text {rec }}\left(V_{\mathrm{OC}}\right)=J_{\mathrm{ph}}\left(V_{\mathrm{OC}}\right) .
$$

Here, the photocurrent is defined as $J_{\mathrm{ph}}\left(V_{\text {int }}\right)=$ $J_{d}\left(V_{\text {int }}\right)-J_{L}\left(V_{\text {int }}\right)$, where $J_{d}\left(V_{\text {int }}\right)$ and $J_{L}\left(V_{\text {int }}\right)$ are the dark and light current at the internal voltage $V_{\text {int }}$, respectively. In order to determine $V_{\mathrm{OC}}$ from Eq. (1), we need a relation between the recombination current and $V$, which is typically assumed to follow a nonideal diode equation

$$
J_{\text {rec }}\left(V_{\text {int }}\right)=J_{0}\left[\exp \left(\frac{q V_{\text {int }}}{n_{\text {id }} k T}\right)-1\right]
$$

where $k$ is Boltzmann's constant and $n_{\text {id }}$ is the ideality factor. For radiative recombination between delocalized states in the conduction and valence band of a semiconductor, the ideality factor $n_{\mathrm{id}}$ is one. At open circuit, there is no current flowing through the external circuit. Thus, series resistance has no effect, and therefore the internal voltage $V_{\text {int }}$ equals the open-circuit voltage $V_{\mathrm{OC}}$. Inserting Eq. (1) in Eq. (2) and solving for $V_{\mathrm{OC}}$, we arrive at

$$
V_{\mathrm{OC}}=\frac{n_{\mathrm{id}} k T}{q} \ln \left(\frac{J_{\mathrm{ph}}\left(V_{\mathrm{OC}}\right)}{J_{0}}+1\right) .
$$

This definition is general for any recombination mechanism controlling $J_{0}$ and $n_{\text {id }}$. For the specific case of radiative recombination, with $J_{0}=J_{0, \text { rad }}$ and $n_{\text {id }}=1$ in Eq. (3), we can write

$$
V_{\mathrm{OC}, \mathrm{rad}}=\frac{k T}{q} \ln \left(\frac{J_{\mathrm{ph}}\left(V_{\mathrm{OC}, \mathrm{rad}}\right)}{J_{0, \mathrm{rad}}}+1\right),
$$

where $J_{\mathrm{ph}}\left(V_{\mathrm{OC}, \text { rad }}\right)$ is the photocurrent at open circuit in the case when there is only radiative recombination.

In the following analysis, we assume that the superposition principle is valid and replace $J_{\mathrm{ph}}\left(V_{\mathrm{OC}}\right)$ by $J_{\mathrm{SC}}$ in Eqs. (3) and (4) [23]. This facilitates the analysis, because the short-circuit current is unaffected by series resistance and so can be measured more accurately than the photocurrent at $V_{\mathrm{OC}}$. In the case when superposition is invalid and $J_{\mathrm{ph}}\left(V_{\mathrm{OC}}\right)$ differs from $J_{\mathrm{SC}}$, the approximation introduces a small error typically of the order of $k T$ in deriving $V_{\text {OC }}$ [24]. In order to determine the radiative limit of the saturation current density $J_{0 \text {,rad, }}$, we need to establish a relation between the emission flux and the applied voltage. In order to do so, we use an InGaAs photodiode array coupled to a grating spectrometer to measure electroluminescence (EL) spectra at various forward biases. However, we will not be able to determine $J_{0 \text {,rad }}$ solely from the EL emission spectrum, because that would require an absolute measurement of the photon density, which is very challenging. Instead, we can make use of detailed balance arguments to calibrate the EL emission using a quantum efficiency measurement as described in the following. 
Under certain specific conditions, in particular, for the case of linear recombination as seen, e.g., in the base of a $p-n$ junction and for the case of radiative recombination between bandlike states (not traps), the EL emission follows an exponential law of internal voltage $V_{\text {int }}$, which is defined as the quasi-Fermi-level splitting at the edge of the space charge region in a $p-n$ junction or Schottky junction. The reciprocity relation [17]

$$
\delta \phi_{\mathrm{em}}(E)=Q_{e}(E) \phi_{\mathrm{BB}}(E)\left[\exp \left(\frac{q V_{\text {int }}}{k T}\right)-1\right]
$$

relates the excess EL emission $\delta \phi_{\mathrm{em}}$ to the internal voltage. The excess EL emission is the total emission minus the blackbody emission $Q_{e}(E) \phi_{\mathrm{BB}}(T)$ of the solar cell in equilibrium. The product $Q_{e}(E) \phi_{\mathrm{BB}}(T)$ is measurable in absolute units using a calibrated quantum efficiency setup, which yields the external solar cell quantum efficiency $Q_{e}$ as a function of energy $E$. The spectral blackbody emission flux density $\phi_{\mathrm{BB}}$ is given by

$$
\phi_{\mathrm{BB}}(E)=\frac{2 \pi E^{2}}{h^{3} c^{2}} \frac{1}{[\exp (E / k T)-1]} \approx \frac{2 \pi E^{2}}{h^{3} c^{2}} \exp \left(\frac{-E}{k T}\right)
$$

in units of photons per area, time, and energy interval. Equation (6) is called a reciprocity relation, because it connects inverse operation modes of a device with each other. In this case, the reciprocity describes the relation between EL emission (light-emitting diode situation) and photocurrent generation (solar cell situation).

Thus, we can determine $J_{0, \text { rad }}$ by integrating over the prefactor of the EL emission in Eq. (5), i.e., via

$$
J_{0, \mathrm{rad}}=q \int_{0}^{\infty} \phi_{\mathrm{em}}(E, V=0) d E=\int_{0}^{\infty} Q_{e}(E) \phi_{\mathrm{BB}}(E) d E
$$

and $V_{\mathrm{OC} \text {,rad }}$ using Eq. (4) with an experimentally determined $J_{\mathrm{SC}}$ (either using a solar simulator or a quantum efficiency setup). This definition of $V_{\mathrm{OC} \text {,rad }}$ actually accounts for the influence of nonradiative recombination on $J_{\mathrm{SC}}$ and so is not truly a radiative limit, but this limit enables a more straightforward definition of the voltage losses. In addition, losses in $J_{\mathrm{SC}}$ due to recombination at short circuit would normally have a small effect on $V_{\mathrm{OC}}$ on $J_{\mathrm{SC}}$. By using the definition of $V_{\mathrm{OC} \text {,rad }}$ and $V_{\mathrm{OC}}$, it can be shown that $[15,17]$

$$
\begin{aligned}
\Delta V_{\mathrm{OC}, \mathrm{nr}} & =V_{\mathrm{OC}, \mathrm{rad}}-V_{\mathrm{OC}}=\frac{k T}{q} \ln \left(\frac{J_{\mathrm{rad}}\left(V_{\mathrm{OC}}\right)}{J_{\mathrm{rec}}\left(V_{\mathrm{OC}}\right)}\right) \\
& =-\frac{k T}{q} \ln \left[Q_{\mathrm{LED}}\left(V_{\mathrm{OC}}\right)\right]
\end{aligned}
$$

where $Q_{\mathrm{LED}}\left(V_{\mathrm{OC}}\right)$ represents the light-emitting diode quantum efficiency $Q_{\mathrm{LED}}$ (of the solar cell) measured at an applied internal voltage equal to the real $V_{\mathrm{OC}}$ of the device. Note that, in Eq. (8), the voltage loss is independent of $J_{\mathrm{SC}}$. This quantity $\left[Q_{\mathrm{LED}}\left(V_{\mathrm{OC}}\right)\right]$ correlates directly with the loss $\Delta V_{\mathrm{OC}, \mathrm{nr}}$ in open-circuit voltage between the radiative limit and the real $V_{\mathrm{OC}}$.

In the SQ theory [1], the quantum efficiency $Q_{e, S Q}$ is defined as a step function

$$
Q_{e, \mathrm{SQ}}(E)=1, \quad E \geq E_{g}, \quad Q_{e, \mathrm{SQ}}(E)=0, \quad E<E_{g} .
$$

By substituting the general quantum efficiency $Q_{e}$ in Eqs. (1)-(7) with the step function $Q_{e, S \mathrm{Q}}$ given by Eq. (9), we can calculate the saturation current density in the SQ limit, $J_{0, \mathrm{SQ}}$, and the SQ open-circuit voltage limit $V_{\mathrm{OC}, \mathrm{SQ}}$. The difference between $V_{\mathrm{OC}, \mathrm{SQ}}$ and the radiative limit $V_{\mathrm{OC} \text {,rad }}$ is primarily due to the fact that, in the SQ limit, the band edge is perfectly abrupt, while the radiative limit [25] can be calculated with an arbitrary band edge that might be smeared out due to disorder or due to the presence of charge transfer absorption. Therefore, the difference between $V_{\mathrm{OC}, \mathrm{SQ}}$ and $V_{\mathrm{OC} \text {,rad }}$ can be used as an indicator of the abruptness of the absorption edge.

\section{RECIPROCITY RELATION IN SILICON AND PEROVSKITE SOLAR CELLS}

Figure 1(a) shows the EL spectrum and the external quantum efficiency of a crystalline Si solar cell (data taken from Ref. [18]). If the EL spectrum is divided by the blackbody spectrum at $T_{c}=300 \mathrm{~K}$, the resulting data set

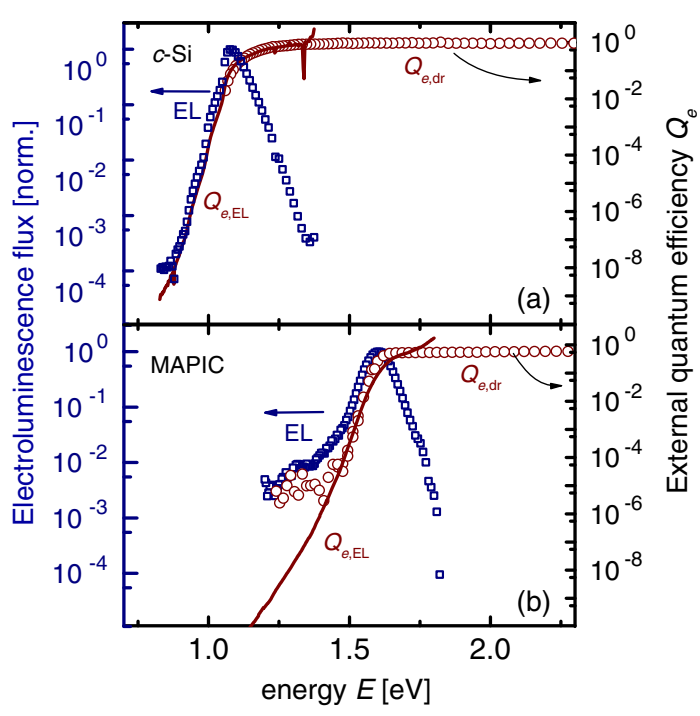

FIG. 1. The external quantum efficiency (open circles), the electroluminescence spectra (open squares), and the quantum efficiency derived from the EL (solid line) for (a) a crystalline silicon solar cell taken from Ref. [18] and (b) a MAPIC device. 
has the same energy dependence as the external quantum efficiency $Q_{e}$. Thus, Eq. (5) does indeed describe the spectral shape of the EL correctly in this case, at least in the range that is experimentally tested in Ref. [18]. Subsequently, Eq. (4) can be used to calculate the radiative open-circuit voltage using the solid line in Fig. 1 that was calibrated by using the directly measured quantum efficiency and the measured $J_{\mathrm{SC}}$. The result for the $\mathrm{Si}$ solar cell in Fig. 1(a) is $V_{\mathrm{OC} \text {,rad }}=864 \mathrm{mV}$, which compares to the actual open-circuit voltage of $V_{\mathrm{OC}}=679 \mathrm{mV}$. The difference between $V_{\mathrm{OC} \text {,rad }}$ and $V_{\mathrm{OC}}$ is due to nonradiative recombination and correlates with the LED quantum efficiency $Q_{\text {LED }}$ in analogy to Eq. (8). This corresponds to a voltage loss $\Delta V_{\mathrm{OC}, \mathrm{nr}}=V_{\mathrm{OC} \text {,rad }}-V_{\mathrm{OC}}=185 \mathrm{mV}$ that is due to radiative recombination being 3 orders of magnitude lower than actual recombination. An external LED quantum efficiency in the range of $10^{-3}=0.1 \%$ is typical for monocrystalline silicon $(c-\mathrm{Si})$ solar cells [19], with the best cells reaching LED quantum efficiencies close to $1 \%$ at temperatures slightly below $300 \mathrm{~K}$ [26].

Figure 1(b) shows the EL spectrum and the external quantum efficiency of a solution-processed methylammonium lead-halide $\mathrm{CH}_{3} \mathrm{NH}_{3} \mathrm{PbI}_{3-x} \mathrm{Cl}_{x}$ (MAPIC) perovskite on mesoporous (mp)- $\mathrm{Al}_{2} \mathrm{O}_{3}$ scaffold solar cell [27-29]. Perovskite thin film solar cells are a recently promoted class of photovoltaic device that have exhibited a rapid growth in performance to reach a confirmed efficiency of $17.9 \%$ [30] and an unconfirmed record of $19.3 \%$ [31] today. However, the efficiency for a single perovskite solar cell can vary greatly under different current-voltage scanning conditions, and a significant hysteresis can be observed. Several hypotheses are proposed to address the origins of the hysteresis [32]. Herein, we discuss the influence of the hysteresis effect on the open-circuit voltage $V_{\mathrm{OC}}$. The MAPIC devices that we study show a high $V_{\mathrm{OC}}$ (about $1.04 \mathrm{~V}$ ) when the voltage is scanned from forward bias (FB) to reverse bias (RB) [33]. When we compare mp- $\mathrm{Al}_{2} \mathrm{O}_{3}$ MAPIC devices to $c$-Si solar cells, the subbandgap quantum efficiency spectrum shows that mp- $\mathrm{Al}_{2} \mathrm{O}_{3}$ MAPIC devices have a blueshifted and broader absorption band edge. A higher-energy absorption onset or a more abrupt absorption edge will lead to a lower-saturation current density given by Eq. (7) and therefore a higher $V_{\mathrm{OC} \text {,rad }}$. In mp- $\mathrm{Al}_{2} \mathrm{O}_{3}$ MAPIC devices, the effects from the higher-energy absorption onset and broadened absorption edge can compensate each other to some extent, but the combined effect of these two mechanisms gives mp- $\mathrm{Al}_{2} \mathrm{O}_{3}$ MAPIC devices a high $V_{\mathrm{OC} \text {,rad }}=1.317 \mathrm{~V}$, which is $453 \mathrm{mV}$ higher than $c$-Si. This value for $V_{\mathrm{OC} \text {,rad }}$ agrees roughly with recently published values by Tress et al. [34]. In mp- $\mathrm{Al}_{2} \mathrm{O}_{3}$ MAPIC devices, the nonradiative voltage loss $\Delta V_{\mathrm{OC}, \mathrm{nr}}$ is $280 \mathrm{mV}$, and the $Q_{\mathrm{LED}}\left(V_{\mathrm{OC}}\right)$ for $\mathrm{mp}-\mathrm{Al}_{2} \mathrm{O}_{3}$ MAPIC devices calculated from Eq. (8) approaches $2 \times 10^{-5}=0.002 \%$. Therefore, nonradiative recombination is dominant in mp- $\mathrm{Al}_{2} \mathrm{O}_{3}$ MAPIC devices, and the
LED quantum efficiency is 500 times lower than the bestperformance crystal silicon device. For different types of perovskite solar cells, there are no observable changes in the EL shape and position at various injection current densities. As shown in Supplemental Material (Sec. VI) [35], this finding implies that the radiative recombination rate at a given voltage stays the same. Therefore, the drop in the open-circuit voltage for different scan conditions and different device structures is related only to changes in nonradiative recombination rates.

\section{RECIPROCITY RELATION IN ORGANIC SOLAR CELLS}

In organic polymer:fullerene blends, typically a weak radiative emission is observed in electroluminescence spectra which is redshifted compared to the emission of the pristine polymer and fullerene films, which is usually assigned to the charge transfer (CT) state at the interface between the polymer and fullerene [5]. These emissive charge transfer states can also be detected in external quantum efficiency spectra with a high dynamic range. Organic molecular semiconductors show a variability in molecular conformation (e.g., a variation of torsional angles between monomers in a polymer chain leading to various conjugation lengths) and in packing (i.e., a variation in the distance between stacked polymer chains) leading to energetic disorder [36-38]. The energetic disorder is sometimes described with Gaussian profiles or exponential tails with typical characteristic energies of the tail $E_{\mathrm{ch}}>k T[6,37]$. While the presence of disorder and localized tail states would allow similar discrepancies from the simple relations given by Eq. (5) as seen for disordered inorganic semiconductors such as amorphous and microcrystalline $\mathrm{Si}$ solar cells [39], the reciprocity relation [Eq. (5)] in polymer:fullerene solar cells is usually still valid $[6,7,40]$.

Figure 2 shows a series of different polymer:fullerene systems that have different polymer LUMO levels and optical band gaps $E_{\text {opt }}$. The optical band gap decreases from $E_{\mathrm{opt}}=1.93 \mathrm{eV}$ for blend devices made with poly(3hexylthiophene) (P3HT) [Fig. 2(a)], via $E_{\text {opt }}=1.61 \mathrm{eV}$ for poly(thieno[3,4-b]thiophene/benzodithiophene) (PTB7) based devices [Fig. 2(b)], to $E_{\text {opt }}=1.31 \mathrm{eV}$ for PDPP3T devices [Fig. 2(c)]. The reduction in the optical band gap leads to a reduction in the energetic difference between the absorption onset $E_{\mathrm{opt}}$ and the CT peak emission but also between $E_{\mathrm{opt}}$ and $q V_{\mathrm{OC}}$. The comparison between Figs. 2(a) and 2(b) summarizes the main developments in polymer development for organic photovoltaics in the past decade. Improvement in solar cell efficiency upon replacing P3HT with PTB7 can be attributed to the depression of both LUMO and HOMO of PTB7 relative to P3HT such that the optical gap is reduced, leading to higher $J_{\mathrm{SC}}$, while at the same time the energy of the CT state is increased, 


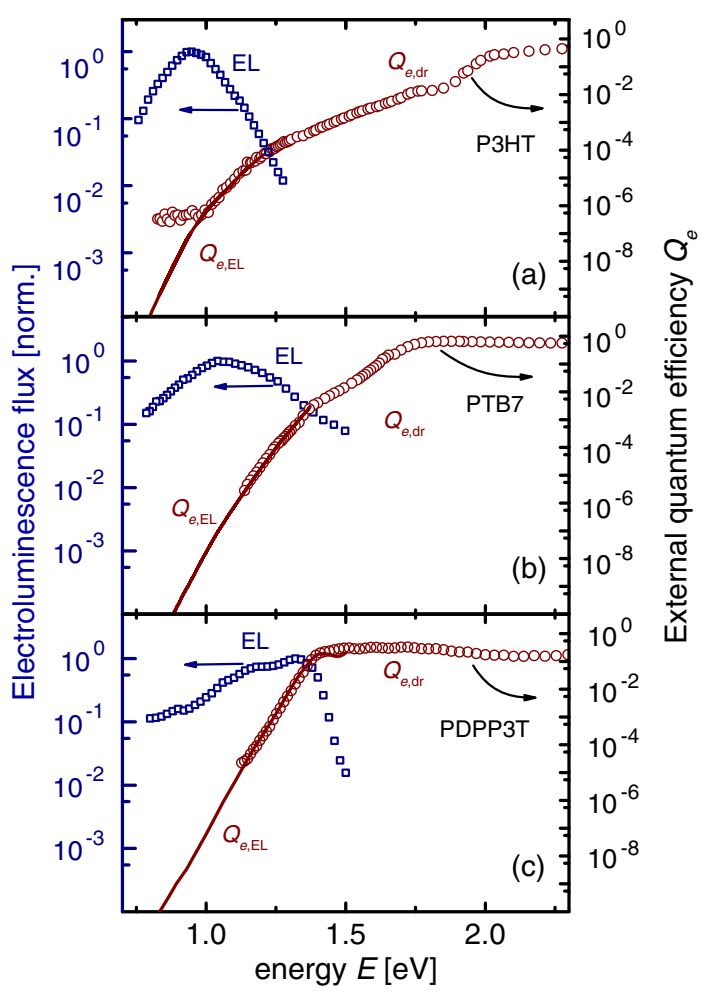

FIG. 2. The external quantum efficiency (open circles), the electroluminescence spectra (open squares), and the quantum efficiency derived from the EL (solid line) for (a) a P3HT: $\mathrm{PC}_{61} \mathrm{BM}$ device, (b) a PTB7:PC ${ }_{71} \mathrm{BM}$ device, and (c) a DPP3T:PC ${ }_{61} \mathrm{BM}$ solar cell.

leading to both a higher EL peak energy and a higher opencircuit voltage. In the case of the low band-gap polymer PDPP3T blended with PCBM [Fig. 2(c)], the difference between $E_{\text {opt }}$ and $E_{\mathrm{EL}}$ has been reduced further to less than $100 \mathrm{meV}$. Here, however, the efficiency is not improved relative to PTB7:PCBM due to the lower photocurrent.

\section{LOSSES IN OPEN-CIRCUIT VOLTAGE FOR DIFFERENT SYSTEMS}

The energetic loss between $E_{\text {opt }}$ and $q V_{\mathrm{OC}}$ in organic heterojunction solar cells can be divided in different ways into components related to distinct loss mechanisms [41,42]. One intuitive way to divide the losses is to use the EL emission peak, representing the CT energy, as determined by using methods as described $[43,44]$ to split the losses between absorption onset and $q V_{\mathrm{OC}}$ into two parts. The part $E_{\mathrm{opt}}-E_{\mathrm{CT}}$ is related to the offset at the heterojunction and intuitively relates to the loss due to exciton dissociation. The second part $E_{\mathrm{CT}}-q V_{\mathrm{OC}}$ would then be related to additional losses due to nongeminate recombination.

The limitation of this way of quantifying energy losses is that the two parts $E_{\mathrm{opt}}-E_{\mathrm{CT}}$ and $E_{\mathrm{CT}}-q V_{\mathrm{OC}}$ cannot be identified directly with physical processes. For instance, the loss $E_{\mathrm{opt}}-E_{\mathrm{CT}}$ also relates to nongeminate recombination rates (in the same way that any change in the band gap in a solar cell affects the recombination rate by changing the equilibrium concentrations of the recombining species of charge carriers). In addition, in the complete absence of any heterojunction offsets and the absence of any nonradiative recombination, the losses do not disappear. Therefore, an alternative and preferable way of quantifying energy and voltage losses in (organic or inorganic) solar cells is to use the detailed balance theory as described in Sec. II. The first loss, $E_{\mathrm{opt}} / q-V_{\mathrm{OC}, \mathrm{SQ}}$, equals roughly $250 \mathrm{mV}$ at typical band gaps relevant for photovoltaics (example $\mathrm{Si}$ : $E_{g}=1.12 \mathrm{eV}, V_{\mathrm{OC}, \mathrm{SQ}}=0.874 \mathrm{~V}$ ). This is comparable to the open-circuit voltage gain $\left[\delta V_{\mathrm{OC}}=k T / q \ln (C)\right]$ available under the maximum possible concentration $C \approx$ 46000 [45]. This substantial loss is unavoidable for any solar cell used without concentration and especially for technologies like organic solar cells that are not intended for concentrator applications. It is frequently disregarded in the description of the maximum $V_{\mathrm{OC}}$ of organic solar cells.

The second loss, $\Delta V_{\mathrm{OC}, \text { abs }}$, is the difference between $V_{\mathrm{OC}, \mathrm{SQ}}$ and $V_{\mathrm{OC} \text {,rad }}$, which is due to replacing the stepfunction-like absorptance of the SQ limit with the actual absorptance of a solar cell material. The $V_{\mathrm{OC} \text {,rad }}$ is then reduced relative to $V_{\mathrm{OC}, \mathrm{SQ}}$, because the emission is redshifted further relative to the absorption edge. In the case of organic solar cells, this loss is relatively large when compared to inorganic solar cells, because the charge transfer state can lie far below the onset of strong absorption, depending on the offsets at the heterojunction. While for $c$-Si, the voltage difference between $V_{\mathrm{OC}, \mathrm{SQ}}$ and $V_{\mathrm{OC}, \text { rad }}$ is only $0.01 \mathrm{~V}$, for $\mathrm{P} 3 \mathrm{HT}: \mathrm{PC}_{61} \mathrm{BM}$, the difference is $0.67 \mathrm{~V}$.

The third loss, $\Delta V_{\mathrm{OC}, \mathrm{nr}}$, is the difference between the radiative open-circuit voltage limit $V_{\mathrm{OC} \text {,rad }}$ and actual opencircuit voltage $V_{\text {OC }}$ [defined in Eq. (9)]. In an actual solar cell, nonradiative recombination is caused by the presence of defects and impurities that act as recombination centers and is enhanced by low mobility.

Table I lists the values of $E_{\mathrm{opt}}$ and $E_{\mathrm{EL}}$ as well as the various $V_{\mathrm{OC}}$ values (SQ limit, radiative limit, and actual measured values) for the set of organic and inorganic devices discussed here. Among all the materials listed in Table I, the inorganic materials all have a relatively low nonradiative voltage loss $\Delta V_{\mathrm{OC}, \mathrm{nr}}$ in the range $0.04-0.21 \mathrm{~V}$. This is partly due to the fact that inorganic materials with large nonradiative recombination losses such as amorphous $\mathrm{Si}$ do not follow the reciprocity relations due to the presence of broad tails of localized states within the band gap and could therefore not be analyzed by using $V_{\mathrm{OC} \text {,rad }}$ as described above [39]. Among the investigated materials, direct crystalline semiconductors with high luminescence efficiency like GaAs have the lowest $\Delta V_{\mathrm{OC}, \mathrm{nr}}$ about $0.04 \mathrm{~V}$. Slightly higher losses are found for materials that are either indirect semiconductors such as monocrystalline silicon (c-Si) or direct but polycrystalline semiconductors such as $\mathrm{Cu}(\mathrm{In}, \mathrm{Ga}) \mathrm{Se}_{2}$ (CIGS) with values in the range of $200 \mathrm{mV}$. 
TABLE I. Voltage loss analysis on different material systems investigated this study. All values are in the unit of volts.

\begin{tabular}{|c|c|c|c|c|c|c|c|c|c|c|}
\hline Material system & $E_{\text {opt }} / q$ & $V_{\mathrm{OC}, \mathrm{SQ}}$ & $V_{\mathrm{OC}, \mathrm{rad}}$ & $V_{\mathrm{OC}}$ & $E_{\mathrm{EL}} / q$ & $E_{\mathrm{opt}} / q-V_{\mathrm{OC}, \mathrm{SQ}}$ & $\Delta V_{\mathrm{OC}, \mathrm{abs}}$ & $\Delta V_{\mathrm{OC}, \mathrm{nr}}$ & $E_{\mathrm{opt}} / q-V_{\mathrm{OC}}$ & $E_{\mathrm{EL}} / q-V_{\mathrm{OC}}$ \\
\hline$c-\mathrm{Si}[18]$ & 1.12 & 0.87 & 0.86 & 0.68 & 0.98 & 0.25 & 0.010 & 0.18 & 0.44 & 0.30 \\
\hline CIGS [18] & 1.18 & 0.96 & 0.95 & 0.74 & 1.18 & 0.22 & 0.011 & 0.21 & 0.44 & 0.44 \\
\hline GaAs [50] & 1.42 & 1.154 & 1.146 & 1.11 & 1.138 & 0.27 & 0.008 & 0.04 & 0.31 & 0.31 \\
\hline MAPI (evap) [51] & 1.61 & 1.332 & 1.330 & 1.08 & 1.60 & 0.28 & 0.002 & 0.25 & 0.53 & 0.52 \\
\hline $\mathrm{mp}-\mathrm{Al}_{2} \mathrm{O}_{3}$ MAPIC (sol p) & 1.61 & 1.33 & 1.32 & 1.04 & 1.60 & 0.28 & 0.01 & 0.28 & 0.57 & 0.56 \\
\hline mp- $\mathrm{TiO}_{2}$ MAPIC (sol p) & 1.61 & 1.33 & 1.32 & 0.84 & 1.60 & 0.28 & 0.01 & 0.48 & 0.77 & 0.76 \\
\hline Inverted MAPI (sol p) & 1.61 & 1.33 & 1.32 & 0.90 & 1.60 & 0.28 & 0.01 & 0.42 & 0.71 & 0.70 \\
\hline $\mathrm{P} 3 \mathrm{HT}: \mathrm{PC}_{61} \mathrm{BM}$ & 1.93 & 1.63 & 0.96 & 0.58 & 0.87 & 0.30 & 0.67 & 0.38 & 1.35 & 0.29 \\
\hline PTB7: PC $_{71}$ BM & 1.61 & 1.33 & 1.13 & 0.74 & 1.08 & 0.28 & 0.20 & 0.39 & 0.87 & 0.34 \\
\hline PDPP3T: $\mathrm{PC}_{61} \mathrm{BM}$ & 1.31 & 1.06 & 1.04 & 0.70 & 1.25 & 0.25 & 0.02 & 0.34 & 0.61 & 0.55 \\
\hline PCDTBT: PC $_{\mathbf{6 1}} \mathrm{BM}$ & 1.82 & 1.53 & 1.25 & 0.90 & 1.21 & 0.29 & 0.28 & 0.35 & 0.92 & 0.31 \\
\hline IDTBT: $\mathrm{PC}_{71} \mathrm{BM}$ & 1.60 & 1.32 & 1.14 & 0.71 & 1.19 & 0.28 & 0.18 & 0.43 & 0.89 & 0.48 \\
\hline BTTDPP: PC $_{71} \mathrm{BM}$ & 1.37 & 1.11 & 1.08 & 0.68 & 1.15 & 0.26 & 0.03 & 0.40 & 0.69 & 0.47 \\
\hline $\mathrm{Si}-\mathrm{PCPDTBT}: \mathrm{PC}_{\mathbf{6 1}} \mathrm{BM}$ & 1.70 & 1.42 & 1.04 & 0.60 & 0.99 & 0.28 & 0.38 & 0.44 & 1.10 & 0.39 \\
\hline $\mathrm{APFO} 3: \mathrm{PC}_{61} \mathrm{BM}$ & 1.88 & 1.59 & 1.38 & 0.99 & 1.34 & 0.29 & 0.21 & 0.39 & 0.89 & 0.35 \\
\hline MDMOPPV : $\mathrm{PC}_{61} \mathrm{BM}$ & 2.15 & 1.83 & 1.22 & 0.85 & 1.22 & 0.32 & 0.61 & 0.37 & 1.30 & 0.37 \\
\hline
\end{tabular}

The mp- $\mathrm{Al}_{2} \mathrm{O}_{3}$ MAPIC solar cell, which possesses a nanocrystalline absorber, has a nonradiative loss slightly higher than $c$-Si and CIGS of about $0.29 \mathrm{~V}$. For the investigated polymer:fullerene systems, the nonradiative voltage losses are relatively high but surprisingly similar in the range of $0.34-0.44 \mathrm{~V}$.

Figures 3 and 4 present the data from Table I graphically for a few representative solar cell materials. Figure 3 uses

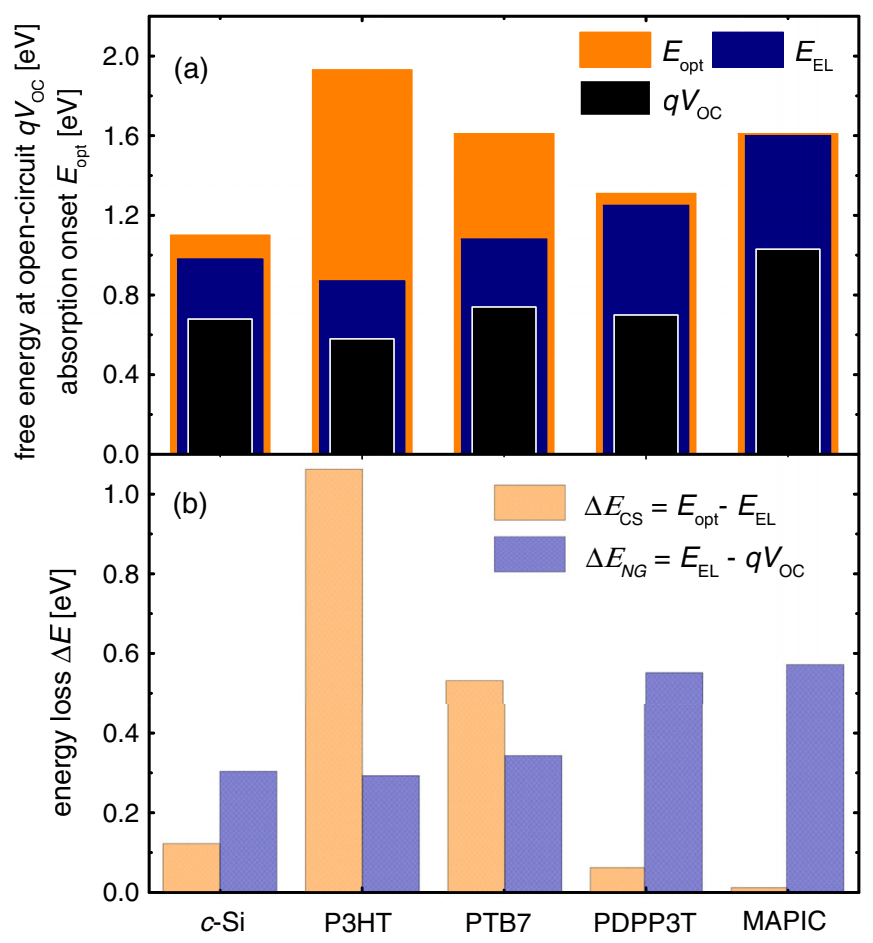

FIG. 3. (a) Comparison of the optical band gap, peak emission of electroluminescence $E_{\mathrm{EL}}$, and actual open-circuit voltage $V_{\mathrm{OC}}$ for crystalline silicon, $\mathrm{P} 3 \mathrm{HT}: \mathrm{PC}_{61} \mathrm{BM}, \quad \mathrm{PTB} 7: \mathrm{PC}_{71} \mathrm{BM}$, PDPP3T: $\mathrm{PC}_{61} \mathrm{BM}$, and a $\mathrm{CH}_{3} \mathrm{NH}_{3} \mathrm{PbI}_{3-x} \mathrm{Cl}_{x}$ (MAPIC). (b) The energetic differences between the quantities plotted in (a). the more intuitive way of splitting the losses using the EL peak, while Fig. 4 shows the data by using $V_{\mathrm{OC}, \mathrm{SQ}}$ and $V_{\text {OC,rad }}$ as reference points.

In Fig. 3, on first sight we observe the expected difference between "excitonic" heterojunction solar cells and classical inorganic solar cells. In the typical organic heterojunction solar cells like P3HT: $\mathrm{PC}_{61} \mathrm{BM}$ [poly(3-hexylthiophene:

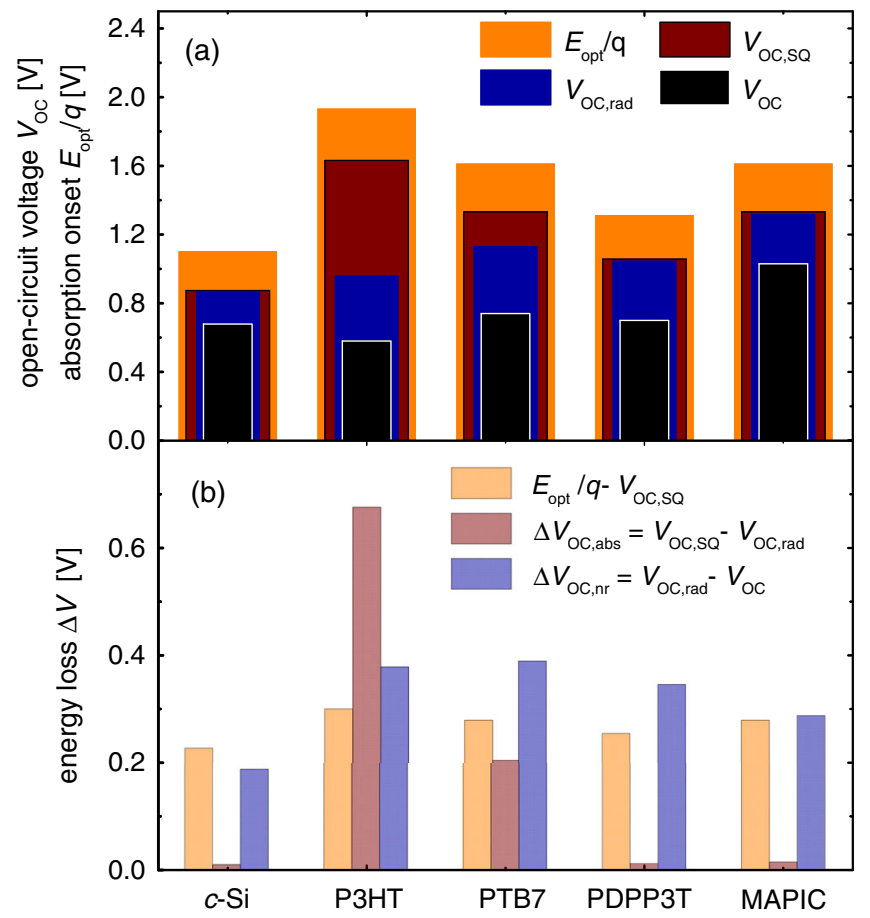

FIG. 4. (a) Comparison of the optical band gap, SQ limit for $V_{\mathrm{OC}}$, radiative open-circuit voltage $V_{\mathrm{OC}, \text { rad }}$, and actual opencircuit voltage $V_{\mathrm{OC}}$ for solar cells with absorbers made from crystalline silicon, $\mathrm{P} 3 \mathrm{HT}: \mathrm{PC}_{61} \mathrm{BM}, \quad \mathrm{PTB} 7: \mathrm{PC}_{71} \mathrm{BM}$, PDPP3T:PC ${ }_{61} \mathrm{BM}$ and a MAPIC. (b) The differences between the quantities plotted in (a). 
[6,6]-phenyl C61-butyric acidmethyl ester] and PTB7: $\mathrm{PC}_{71} \mathrm{BM}[22,27]$, the bar for the difference $\Delta E_{\mathrm{CS}}=$ $E_{\mathrm{opt}}-E_{\mathrm{EL}}$ is huge compared to the case of inorganic solar cells, where this loss is negligible. Perovskite solar cells (MAPIC) behave clearly like inorganic solar cells. Because of the abruptness of the MAPIC band gap, the loss $\Delta E_{\mathrm{CS}}$ is even smaller than for the indirect semiconductor Si. However, there is also one polymer:fullerene blend (namely, PDPP3T: $\mathrm{PC}_{61} \mathrm{BM}$ ) that—using these metrics — behaves like an inorganic semiconductor. Here, the EL peak is even closer to the absorption onset than for crystalline $\mathrm{Si}$. While PDPP3T: $\mathrm{PC}_{61} \mathrm{BM}$ is known to be the polymer:fullerene blend with the smallest total difference $E_{\text {opt }}-q V_{\mathrm{OC}}$, it appears to have a comparably large amount of nongeminate recombination losses. The same seems to hold true for MAPIC. For the material systems we investigate here, the EL peak position shows no or only a negligible shift with bias voltage. Therefore, the EL emission does not seem to be affected by the filling of band tail states [6]. Thus, the comparison between EL emission from various materials is valid. When using the detailed balance theory, the situation is slightly different. Figure 4 shows that, while PDPP3T: $\mathrm{PC}_{61} \mathrm{BM}$ still has the smallest losses in terms of $V_{\mathrm{OC}, \mathrm{SQ}}-V_{\mathrm{OC}, \text { rad }}$, also the losses due to nonradiative recombination $(0.34 \mathrm{~V})$ are smaller as compared to $\mathrm{P} 3 \mathrm{HT}$ and PTB7. This is consistent with the fact that PDPP3T: $\mathrm{PC}_{61} \mathrm{BM}$ has reasonable fill factors of 0.67 [21] and a reasonable charge carrier lifetime [46-48]. Also in the case of mp- $\mathrm{Al}_{2} \mathrm{O}_{3}$ MAPIC, we see that the losses due to nonradiative recombination are clearly not exceeding those of typical organic solar cell materials as one might have guessed from Fig. 3.

Among the three polymer:fullerene systems, PTB7, the polymer with the intermediate LUMO-LUMO offset, has the highest efficiency of $9.2 \%$ reported by He et al. [22]. For P3HT: $\mathrm{PC}_{61} \mathrm{BM}$, the LUMO-LUMO offset is too large, resulting in a big voltage loss between the optical band gap and $V_{\mathrm{OC} \text {,rad }}$, while for PDPP3T, the energetic offset is less than $100 \mathrm{meV}$. Although PDPP3T has improved voltage losses relative to other organic systems, it shows relatively low photocurrent quantum efficiency leading to similar power conversion efficiency to $\mathrm{P} 3 \mathrm{HT}: \mathrm{PC}_{61} \mathrm{BM}$. PTB7 is one of the polymers with the highest efficiency published to date, and it benefits from a LUMO-LUMO offset that results in a good compromise between charge separation and $V_{\mathrm{OC}}$. For the case of P3HT: PCBM, as shown in Fig. 2, we observe a large voltage loss $\Delta V_{\mathrm{OC} \text {,abs }}$ due to the large offset between the EL peak position and polymer absorption onset. In Supplemental Material (Sec. V) [49], we show how to further split this offset into contributions due to the difference between absorption onset and CT state and additional losses due to the smeared absorption edge of the polymer. As expected, the loss of $670 \mathrm{mV}$ largely originates from the energy difference between absorption onset and CT state $(630 \mathrm{mV})$ and only to a small degree from the smeared absorption onset of the polymer $(40 \mathrm{mV})$.
So far, we discussed only the open-circuit voltage of different solar cell technologies and how the reduction of $V_{\mathrm{OC}}$ relative to its thermodynamic limit can be understood. It is worthwhile to test whether there is a relation between the losses discussed so far and the efficiency of the solar cells rather than only the $V_{\mathrm{OC}}$. The most important loss mechanism discussed so far is the loss due to nonradiative recombination. This loss will affect not only $V_{\mathrm{OC}}$ but also the $F F$ and $J_{\mathrm{SC}}$ of solar cells, and therefore it should correlate with efficiency. If a solar cell was close to its optimum, then we would expect charge collection to be efficient and the $J_{\mathrm{SC}}$ and $F F$ therefore to be quite close to their limit. The main loss should then be due to the $V_{\mathrm{OC}}$ being reduced relative to the thermodynamic limit. As defined by Eq. (8), the nonradiative loss can also be expressed by using the LED quantum efficiency $Q_{\mathrm{LED}}$.

Therefore, we plot in Fig. 5 the efficiency normalized to the SQ limit versus the logarithm of the LED quantum efficiency, $Q_{\mathrm{LED}}$, which is proportional to the voltage loss due to nonradiative recombination. We plot a number of $Q_{\mathrm{LED}}$ and normalized PCE values from OPV, perovskite, and inorganic solar cells. The values for $Q_{\mathrm{LED}}$ obtained for different solar cell technologies lie in the range $10^{-9}$ to 0.22 and the corresponding nonradiative voltage losses are

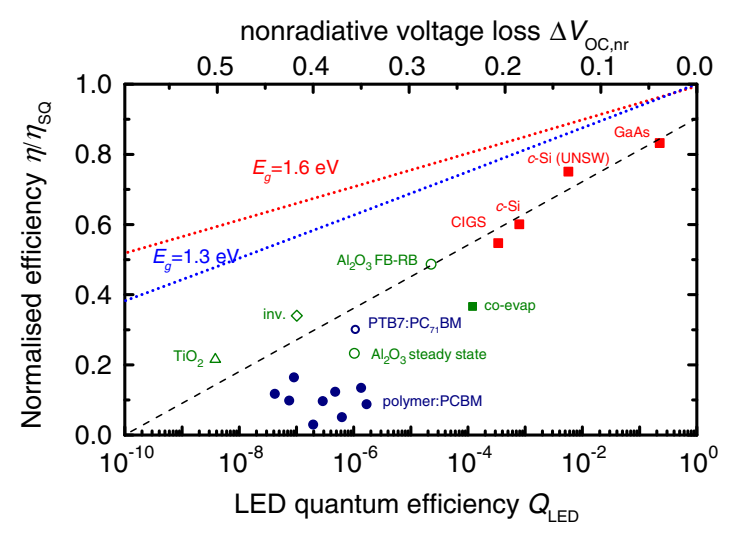

FIG. 5. Power conversion efficiency for different solar cell technologies normalized to the Shockley-Queisser limits as a function of LED quantum efficiency $Q_{\text {LED }}$ both taken from literature and collected in this work. The dotted lines define the theoretical limits of various $Q_{\mathrm{LED}}$ at two different optical band gaps: 1.6 and $1.3 \mathrm{eV}$. The top $x$ axis is the nonradiative voltage loss over the range of $Q_{\mathrm{LED}}$, referring to Eq. (8). The data points for inorganic solar cells are shown in red squares. Different perovskite fabrication technologies are shown in green. Open green and circle points represents solution-processed perovskite devices made with MAPIC on mp- $\mathrm{TiO}_{2}$ and $\mathrm{mp}-\mathrm{Al}_{2} \mathrm{O}_{3}$ films, respectively. The open green diamond point is the solutionprocessed inverted MAPI device using PEDOT:PSS and PCBM interlayers. The solid square point is the coevaporated MAPI taken from Ref. [40]. Different organic solar cells are shown in blue. PTB7: $\mathrm{PC}_{71} \mathrm{BM}$, the OPV system with the highest PCE, is shown as the open blue circle. The dashed line is a guide to the eye representing the approximate experimental trend. 
shown on the top $x$ axis, referring to Eq. (8). The values for the inorganic solar cells are taken from the list summarized by Green [50] and from Ref. [18], respectively. The data for organic solar cells are collected in this work and listed in Table I. Values for an evaporated perovskite solar cell are taken from a recent publication by Tvingstedt et al. [51]. The absolute value of $Q_{\mathrm{LED}}$ for thermal-evaporated $\mathrm{CH}_{3} \mathrm{NH}_{3} \mathrm{PbI}_{3}$ (MAPI) samples is experimentally measured. The $Q_{\mathrm{LED}}$ values for the GaAs device from Green's work [50] and the other systems reported by us are derived from the spectra of EL and EQE combined with the actual opencircuit voltage using Eq. (8). In terms of accuracy of calculated voltage losses, the voltage-dependent photocurrent can lead to small errors on the order of $k T$, and estimation of errors is discussed in more detail in Supplemental Material (Sec. IV)[24]. We also show perovskite devices fabricated by three different solution-process based routes. Of these, the device made by single step deposition on mesoporous- $\mathrm{TiO}_{2}$ [27] and the device made with PEDOT:PSS and PCBM interlayers in an inverted structure show little hysteresis in current-voltage $(J V)$ measurements. Therefore, for these two cases, we show only the data taken from steady-state $J V$ scanning conditions in Fig. 5. For the third type of perovskite device, made by single step deposition on mesoporous- $\mathrm{Al}_{2} \mathrm{O}_{3}$, strong hysteresis is seen in the $J V$ measurement. Therefore, two data points taken under forward to reverse bias (FB-RB) scan and steady-state condition are plotted in Fig. 5 for this case. Details of current-voltage $(J V)$ measurements of perovskite solar cells, including the "steady-state" condition, are provided in Supplemental Material, and more detailed measurement results are found in Fig. S5 [33].

The data points in Fig. 5 show the expected general trend that high $Q_{\mathrm{LED}}$ values also lead to high normalized efficiencies. A slightly surprising factor is that the normalized efficiencies of inorganic, solution-processed MAPI and MAPIC perovskite, and the best OPV solar cells show a simple, approximately linear relation to $\ln \left(Q_{\mathrm{LED}}\right)$, shown as the dashed line in Fig. 5. This linear relation between efficiency and $\ln \left(Q_{\mathrm{LED}}\right)$ indicates that, for mature inorganic systems and for the best OPV solar cells, efficiency could be increased by increasing $Q_{\mathrm{LED}}$. Any change in $Q_{\mathrm{LED}}$ has two direct effects on efficiency. It directly correlates with $V_{\text {OC }}$ via Eq. (4), and any reduction in $V_{\text {OC }}$ will reduce the maximum available $F F$ [52]. Thus, one can predict the effect of $Q_{\mathrm{LED}}$ on the power conversion efficiency by just taking changes in $V_{\mathrm{OC}}$ and $F F$ into account. These predictions are shown by the dotted lines in Fig. 5 for two representative band gaps. It is obvious that the empirical trend features additional losses in efficiency that are not captured by that approach. These include charge collection losses, series resistance, and reflection losses that will further reduce the $F F$ and the short-circuit current. These losses may cause the empirical result to be only $90 \%$ of the SQ limit at $Q_{\mathrm{LED}}=1$. Within the group of organic solar cells, the LED quantum efficiency is not a good indicator of quality, which means that here nonradiative recombination is not the only problem, but instead variations in the losses, e.g., due to the smeared-out absorption edge (i.e., variations in $V_{\mathrm{OC}, \mathrm{SQ}}-V_{\mathrm{OC} \text {,rad }}$ ) or due to geminate recombination, are more dominant as seen also in Fig. 4(b). PDPP3T: $\mathrm{PC}_{61} \mathrm{BM}$ is again a good example, because it should, in general, be a very promising material showing low voltage losses in every respect, but it still fails to achieve efficiencies $>4 \%$ because of the low $Q_{e}$ at short circuit. This analysis suggests that the low $Q_{e}$ results from factors other than nonradiative recombination, for example, geminate recombination.

Compared to the theoretical lines (dotted), the empirical line (dashed) is lower in magnitude with a steeper gradient. For the technologies on the empirical line with higher $Q_{\mathrm{LED}}$ values, such as GaAs and $c$-Si solar cells, the efficiencies deviate less from the theoretical value, and these photovoltaic technologies tend to be commercially mature. However, new technologies, such as OPV and perovskites, have lower $Q_{\mathrm{LED}}$ values, and the efficiencies are often substantially below the theoretical limits. The low $Q_{\text {LED }}$ indicates that OPV and perovskites still suffer from higher nonradiative recombination rates than the shown inorganic solar cells. The large offset between the theoretical and empirical efficiencies implies that these new and less mature technologies also suffer large losses from other factors such as charge collection, series resistance, parasitic absorption, and reflection, which are not distinguished in this analysis.

\section{CONCLUSION}

In summary, we discuss luminescence-based methods to analyze voltage losses in solution-processed solar cells and provide an overview over the data obtained from a series of organic and perovskite solar cells that we compare with published data on classical inorganic solar cells. In order to explain which physical mechanism reduces the open-circuit voltage relative to its thermodynamic limits, we use different reference points, namely, the open-circuit voltage $V_{\mathrm{OC}, \mathrm{SQ}}$ in the SQ limit (step-function-like absorptance and radiative recombination only) and the radiative opencircuit voltage (arbitrary absorptance and radiative recombination only). This analysis is useful to understand the voltage losses in an actual solar cell, as the voltage differences between the reference points and actual $V_{\mathrm{OC}}$ can be attributed to specific physical mechanisms. The loss between $V_{\mathrm{OC}, \mathrm{SQ}}$ and $V_{\mathrm{OC}, \text { rad }}$ is due to the smeared-out absorption edge and depends, for instance, on the amount of disorder in a semiconductor or on the LUMO-LUMO offset at the donor-acceptor heterointerface. The difference between $V_{\mathrm{OC} \text {,rad }}$ and $V_{\mathrm{OC}}$ is caused by nonradiative recombination and is directly related to the LED quantum efficiency of the solar cell. In comparison with state-ofthe-art inorganic and MAPIC solar cells, many organic solar 
cells show a high voltage loss due to the broadened absorption edge. However, there exist some materials such as PDPP3T: $\mathrm{PC}_{61} \mathrm{BM}$ that have voltage losses that are comparable to the losses in inorganic or perovskite devices. However, currently the materials with the lowest losses in $V_{\mathrm{OC}}$ do not show the highest efficiencies. Therefore, we need to understand those materials and establish whether it is possible to combine minimum losses due to the heterojunction and due to nonradiative recombination with high external quantum efficiencies. We herein also highlight an empirical linear relation between the efficiency normalized to the Shockley-Queisser limit and the logarithm of the LED quantum efficiency in different photovoltaic technologies.

\section{ACKNOWLEDGMENTS}

The authors thank Dr. Xiaoe Li, Dr. Pabitra Tuladhar, and Professor James Durrant for fabricating inverted perovskite devices. J. Y. thanks Imperial College London Rector's Scholarship Fund (Ph.D.). W. G. acknowledges support from People's Republic of China's State Scholarship Fund. T. K. acknowledges support by an Imperial College Junior Research Fellowship, support from the DFG (Grant No. KI-1571/2-1) and from the Helmholtz Association via the Helmholtz Energy Alliance Hybrid PV. J. N. acknowledges The Royal Society for a Wolfson Merit Award and the Engineering and Physical Sciences Research Council for support via Grants No. EP/K029843/1, No. EP/ K030671/1, and No. EP/J017361/1.

[1] W. Shockley and H. J. Queisser, Detailed balance limit of efficiency of $p$ - $n$ junction solar cells, J. Appl. Phys. 32, 510 (1961).

[2] T. Markvart, Solar cell as a heat engine: Energy-entropy analysis of photovoltaic conversion, Phys. Status Solidi A 205, 2752 (2008).

[3] L. C. Hirst and N. J. Ekins-Daukes, Fundamental losses in solar cells, Prog. Photovoltaics 19, 286 (2011).

[4] $250 \mathrm{meV}$ is correct for a band gap $E_{g}$ of about $1.18 \mathrm{eV}$. For higher (lower) band gaps, the difference $E_{g}-q V_{\mathrm{OC}, \mathrm{SQ}}$ becomes larger (smaller) by about $6 \mathrm{meV}$ per $100 \mathrm{meV}$ change in band gap.

[5] K. Tvingstedt, K. Vandewal, A. Gadisa, F. L. Zhang, J. Manca, and O. Inganas, Electroluminescence from charge transfer states in polymer solarcells, J. Am. Chem. Soc. 131, 11819 (2009).

[6] W. Gong, M. A. Faist, N. J. Ekins-Daukes, Z. Xu, D. D. C. Bradley, J. Nelson, and T. Kirchartz, Influence of energetic disorder on electroluminescence emission in polymer: fullerene solar cells, Phys. Rev. B 86, 024201 (2012).

[7] K. Vandewal, K. Tvingstedt, A. Gadisa, O. Inganas, and J. V. Manca, On the origin of the open-circuit voltage of polymer-fullerene solar cells, Nat. Mater. 8, 904 (2009).

[8] P. K. Nayak, J. Bisquert, and D. Cahen, Assessing possibilities and limits for solar cells, Adv. Mater. 23, 2870 (2011).
[9] P. K. Nayak, G. Garcia-Belmonte, A. Kahn, J. Bisquert, and D. Cahen, Photovoltaic efficiency limits and material disorder, Energy Environ. Sci. 5, 6022 (2012).

[10] P. K. Nayak and D. Cahen, Updated assessment of possibilities and limits for solar cells, Adv. Mater. 26, 1622 (2014).

[11] T. Kirchartz and U. Rau, Electroluminescence analysis of high efficiency $\mathrm{Cu}(\mathrm{In}, \mathrm{Ga}) \mathrm{Se}_{2}$ solar cells, J. Appl. Phys. 102, 104510 (2007).

[12] K. Vandewal, Z. Ma, J. Bergqvist, Z. Tang, E. Wang, P. Henriksson, K. Tvingstedt, M. R. Andersson, F. Zhang, and O. Inganäs, Quantification of quantum efficiency and energy losses in low bandgap polymer:fullerene solar cells with high open-circuit voltage, Adv. Funct. Mater. 22, 3480 (2012).

[13] M. A. Faist, T. Kirchartz, W. Gong, R. S. Ashraf, I. McCulloch, J. C. de Mello, N. J. Ekins-Daukes, D. D. C. Bradley, and J. Nelson, Competition between the charge transfer state and the singlet states of donor or acceptor limiting the efficiency in polymer:fullerene solar cells, J. Am. Chem. Soc. 134, 685 (2012).

[14] K. Vandewal, A. Gadisa, W. D. Oosterbaan, S. Bertho, F. Banishoeib, I. Van Severen, L. Lutsen, T. J. Cleij, D. Vanderzande, and J. V. Manca, The relation between opencircuit voltage and the onset of photocurrent generation by charge-transfer absorption in polymer:fullerene bulk heterojunction solar cells, Adv. Funct. Mater. 18, 2064 (2008).

[15] R. T. Ross, Some thermodynamics of photochemical systems, J. Chem. Phys. 46, 4590 (1967).

[16] G. Smestad and H. Ries, Luminescence and current voltage characteristics of solar-cells and optoelectronic devices, Sol. Energy Mater. Sol. Cells 25, 51 (1992).

[17] U. Rau, Reciprocity relation between photovoltaic quantum efficiency and electroluminescent emission of solar cells, Phys. Rev. B 76, 085303 (2007).

[18] T. Kirchartz, U. Rau, M. Kurth, J. Mattheis, and J. H. Werner, Comparative study of electroluminescence from $\mathrm{Cu}(\mathrm{In}, \mathrm{Ga}) \mathrm{Se}_{2}$ and Si solar cells, Thin Solid Films 515, 6238 (2007).

[19] T. Kirchartz, A. Helbig, W. Reetz, M. Reuter, J. H. Werner, and U. Rau, Reciprocity between electroluminescence and quantum efficiency used for the characterization of silicon solar cells, Prog. Photovoltaics 17, 394 (2009).

[20] L. Koster, S. E. Shaheen, and J. C. Hummelen, Pathways to a new efficiency regime for organic solar cells, Adv. Energy Mater. 2, 1246 (2012).

[21] J. C. Bijleveld, A. P. Zoombelt, S. G. J. Mathijssen, M. M. Wienk, M. Turbiez, D. M. de Leeuw, and R. A. J. Janssen, Poly(diketopyrrolopyrrole-terthiophene) for ambipolar logic and photovoltaics, J. Am. Chem. Soc. 131, 16616 (2009).

[22] Z. He, C. Zhong, S. Su, M. Xu, H. Wu, and Y. Cao, Enhanced power-conversion efficiency in polymer solar cells using an inverted device structure, Nat. Photonics 6, 591 (2012).

[23] F. A. Lindholm, J. G. Fossum, and E. L. Burgess, Application of the superposition principle to solar-cell analysis, IEEE Trans. Electron Devices 26, 165 (1979).

[24] See Supplemental Material at http://link.aps.org/ supplemental/10.1103/PhysRevApplied.4.014020 for an 
analysis of the influence of violating the superposition principle on Eqs. (4) and (8).

[25] T. Tiedje, E. Yablonovitch, G. D. Cody, and B. G. Brooks, Limiting efficiency of silicon solar cells, IEEE Trans. Electron Devices 31, 711 (1984).

[26] M. A. Green, J. H. Zhao, A. H. Wang, P. J. Reece, and M. Gal, Efficient silicon light-emitting diodes, Nature (London) 412, 805 (2001).

[27] See Supplemental Material at http://link.aps.org/ supplemental/10.1103/PhysRevApplied.4.014020 for device fabrication details.

[28] N. J. Jeon, J. H. Noh, Y. C. Kim, W. S. Yang, S. Ryu, and S. I. Seok, Solvent engineering for high-performance inorganic-organic hybrid perovskite solar cells, Nat. Mater. 13, 897 (2014).

[29] H. S. Kim et al., Lead iodide perovskite sensitized all-solidstate submicron thin film mesoscopic solar cell with efficiency exceeding 9\%, Sci. Rep. 2, 591 (2012).

[30] M. A. Green, K. Emery, Y. Hishikawa, W. Warta, and E. D. Dunlop, Solar cell efficiency tables (version 44), Prog. Photovoltaics 22, 701 (2014).

[31] H. Zhou, Q. Chen, G. Li, S. Luo, T.-b. Song, H.-S. Duan, Z. Hong, J. You, Y. Liu, and Y. Yang, Interface engineering of highly efficient perovskite solar cells, Science 345, 542 (2014).

[32] H. J. Snaith, A. Abate, J. M. Ball, G. E. Eperon, T. Leijtens, N. K. Noel, S. D. Stranks, J. Tse-Wei Wang, K. Wojciechowski, and W. Zhang, Anomalous hysteresis in perovskite solar cells, J. Phys. Chem. Lett. 5, 1511 (2014).

[33] See Supplemental Material at http://link.aps.org/ supplemental/10.1103/PhysRevApplied.4.014020 for perovskite solar cell $J V$ measurements.

[34] W. Tress, N. Marinova, O. Inganas, M. Nazeeruddin, S. M. Zakeeruddin, and M. Graetzel, Predicting the open-circuit voltage of $\mathrm{CH}_{3} \mathrm{NH}_{3} \mathrm{PbI}_{3}$ perovskite solar cells using electroluminescence and photovoltaic quantum efficiency spectra: The role of radiative and non-radiative recombination, Adv. Energy Mater. 5, 00812 (2014).

[35] See Supplemental Material at http://link.aps.org/ supplemental/10.1103/PhysRevApplied.4.014020 for radiative recombination rates under various voltage biases.

[36] J. Rivnay, R. Noriega, J. E. Northrup, R. J. Kline, M. F. Toney, and A. Salleo, Structural origin of gap states in semicrystalline polymers and the implications for charge transport, Phys. Rev. B 83, 121306 (2011).

[37] R. A. Street, K. W. Song, J. E. Northrup, and S. Cowan, Photoconductivity measurements of the electronic structure of organic solar cells, Phys. Rev. B 83, 165207 (2011).
[38] J. M. Frost, J. Kirkpatrick, T. Kirchartz, and J. Nelson, Parameter free calculation of the subgap density of states in poly (3-hexylthiophene), Faraday Discuss. 174, 255 (2014).

[39] T. C. M. Müller, B. E. Pieters, T. Kirchartz, R. Carius, and U. Rau, Effect of localized states on the reciprocity between quantum efficiency and electroluminescence in $\mathrm{Cu}(\mathrm{In}, \mathrm{Ga}) \mathrm{Se}_{2}$ and $\mathrm{Si}$ thin-film solar cells, Sol. Energy Mater. Sol. Cells 129, 95 (2014).

[40] K. Vandewal et al., Efficient charge generation by relaxed charge-transfer states at organic interfaces, Nat. Mater. 13, 63 (2014).

[41] U. Rau, U. W. Paetzold, and T. Kirchartz, Thermodynamics of light management in photovoltaic devices, Phys. Rev. B 90, 035211 (2014).

[42] U. Rau and T. Kirchartz, On the thermodynamics of light trapping in solar cells, Nat. Mater. 13, 103 (2014).

[43] K. Vandewal, K. Tvingstedt, A. Gadisa, O. Inganas, and J. V. Manca, Relating the open-circuit voltage to interface molecular properties of donor:acceptor bulk heterojunction solar cells, Phys. Rev. B 81, 125204 (2010).

[44] R. A. Street, S. A. Hawks, P. P. Khlyabich, G. Li, B. J. Schwartz, B. C. Thompson, and Y. Yang, Electronic structure and transition energies in polymer fullerene bulk heterojunctions, J. Phys. Chem. C 118, 21873 (2014).

[45] J. Nelson, The Physics of Solar Cells (Imperial College Press, London, 2003), p. 263.

[46] See Supplemental Material at http://link.aps.org/ supplemental/10.1103/PhysRevApplied.4.014020 for a comparison of the charge carrier lifetimes of different polymer-fullerene blends.

[47] D. Credgington and J. R. Durrant, Insights from transient optoelectronic analyses on the open-circuit voltage of organic solar cells, J. Phys. Chem. Lett. 3, 1465 (2012).

[48] F. Deledalle, Ph.D. thesis, Imperial College, London, 2014.

[49] See Supplemental Material at http://link.aps.org/ supplemental/10.1103/PhysRevApplied.4.014020 for estimation of voltage losses from polymer tail states and CT states.

[50] M. A. Green, Radiative efficiency of state-of-the-art photovoltaic cells, Prog. Photovoltaics 20, 472 (2012).

[51] K. Tvingstedt, O. Malinkiewicz, A. Baumann, C. Deibel, H. J. Snaith, V. Dyakonov, and H. J. Bolink, Radiative efficiency of lead iodide based perovskite solar cells, Sci. Rep. 4, 6071 (2014).

[52] M. A. Green, Accuracy of analytical expressions for solar cell fill factors, Sol. Cells 7, 337 (1982). 